\title{
Fatores facilitadores e dificultadores da notificação de eventos adversos: revisão integrativa
}

Facilitating and hindering factors for reporting adverse events: an integrative review Factores facilitadores y dificultades en la notificación de eventos adversos: revisión integradora

\author{
Saimon da Silva Nazário ${ }^{1}$ ib https://orcid.org//0000-0001-7971-7765 \\ Elaine Drehmer de Almeida Cruz' 1 ib https://orcid.org/10000-0002-7686-6340 \\ Robson Giovani Paes' ${ }^{1}$ ib https://orcid.org/0000-0001-6899-4054 \\ Maria de Fátima Mantovani' io https://orcid.org/0000-0001-6899-4054 \\ Leila Soares Seiffert ${ }^{2}$ io https://orcid.org/0000-0002-8461-0361
}

Como citar:

Nazário SS, Cruz ED, Paes RG, Mantovani

MF, Seiffert LS. Fatores facilitadores e

dificultadores da notificação de eventos adversos: revisão integrativa. Acta Paul Enferm. 2021;34:AAPE001245.

DOI

http://dx.doi.org/10.37689/actaape/2021AR01245

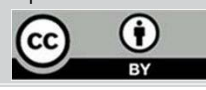

Descritores medicação; Notificação; Gestão de riscos

Keywords

Patient safety; Medical errors; Medication errors: Notification; Risk management

Descriptores

Seguridad del paciente; Errores médicos; Errores de medicación; Notificación; Gestión de riesgos

Submetido

1 de Junho de 2020

Aceito

1 de Março de 2021

Autor correspondente

Saimon da Silva Nazário

E-mail: nazariosaimon2@gmail.com

\section{Resumo}

Objetivo: Analisar as evidências disponíveis na literatura acerca dos fatores dificultadores e facilitadores para a notificação de eventos adversos.

Métodos: Revisão integrativa da literatura, com a questão norteadora embasada no acrônimo PCC (Problema, Conceito e Contexto): quais os fatores facilitadores e dificultadores que interferem na notificação de eventos adversos em serviços de saúde? Utilizou-se as diretrizes The Preferred Reporting Items for Systematic Reviews and Meta-Analysis como guia para reportar o método de revisão; os critérios de inclusão foram publicações de estudos primários entre 2015 e 2019, nos idiomas português e/ou espanhol e/ou inglês. A busca das publicações ocorreu no mês de março de 2020, na Biblioteca Regional Virtual de Saúde, com a estratégia "erros médicos" OR "erros de medicação" AND "notificação" e na National Library of Medicine e SCOPUS com "risk management" OR "patient safety" AND "mandatory reporting".

Resultados: Foram encontrados 2195 estudos, dos quais 31 eram elegíveis; após a leitura na íntegra 11 compuseram a amostra final. Os fatores foram agrupados, sendo os facilitadores: apoio institucional aos profissionais; cultura de segurança organizacional; aprimoramento do sistema de notificação e incentivo ao relato voluntário e confidencial. Os dificultadores: falta de recursos materiais/humanos; medo/vergonha; postura institucional punitiva/falta de amparo; falta de estímulo à notificação e lacunas no conhecimento.

Conclusão: A síntese desses fatores pode ser utilizada para otimizar medidas assistenciais e gerenciais com provimento de recursos materiais, pessoais, capacitação e promoção da cultura de segurança, com vistas ao incentivo a notificação, buscando indicadores fidedignos desses agravos.

\section{Abstract}

Objective: To analyze the evidence available in scientific literature about hindering and facilitating factors for adverse event reporting.

Methods: This is an integrative literature review, with the guiding question based on the acronym PCC (Problem, Concept and Context): which factors facilitate and hinder that interfere with adverse event reporting in health services? The Preferred Reporting Items for Systematic Reviews and Meta-Analysis was used as a guide to report the review method; inclusion criteria were publications of primary studies between 2015 and 2019, in Brazilian Portuguese and/or Spanish and/or English. Publication search took place in March 2020 at the Virtual Regional Health Library, using "erros médicos" (medical errors) OR "erros de medicação" (medication errors) AND "notificação" (reporting), and in the National Library of Medicine and SCOPUS, using "risk management" OR "safety patient" AND "mandatory reporting". 
Results: A total of 2,195 studies was found, of which 31 were eligible; after reading in full, 11 comprised the final sample. The facilitating factors were grouped, namely: institutional support to professionals; organizational safety culture; reporting system improvement; incentive to voluntary and confidential report. The hindering factors: lack of material/human resources; fear/shame; punitive institutional posture/lack of protection; lack of encouragement to reporting; gaps in knowledge.

Conclusion: The synthesis of these factors can be used to optimize care and management measures with the provision of material, personal resources, training and promotion of a safety culture, with a view to encouraging reporting, seeking reliable indicators of these injuries.

\section{Resumen}

Objetivo: Analizar las evidencias disponibles en la literatura acerca de los factores facilitadores y dificultades para la notificación de eventos adversos.

Métodos: Revisión integradora de la literatura, con la pregunta orientadora basada en el acrónimo PCC (problema, concepto y contexto): ¿Cuáles son los factores facilitadores y las dificultades que interfieren en la notificación de eventos adversos en los servicios de salud? Se utilizaron las directrices The Preferred Reporting Items for Systematic Reviews and Meta-Analysis como guía para reportar el método de revisión. Los criterios de inclusión fueron publicaciones de estudios primarios entre 2015 y 2019, en idioma portugués, español o inglés. La búsqueda de las publicaciones se llevó a cabo en el mes de marzo de 2020, en la Biblioteca Regional Virtual de Salud, con la estrategia "errores médicos" OR "errores de medicación" AND "notificación" y en la National Library of Medicine y SCOPUS con "risk management" OR "patient safety" AND "mandatory reporting".

Resultados: Se encontraron 2.195 estudios, de los cuales 31 eran elegibles. Luego de la lectura completa, 11 formaron la muestra final. Los factores fueron agrupados en dos, los facilitadores: apoyo institucional a los profesionales, cultura de seguridad organizacional, mejora del sistema de notificación e incentivo al relato voluntario y confidencial. Y las dificultades: falta de recursos materiales/humanos, miedo/vergüenza, postura institucional punitiva/falta de amparo, falta de estímulo para la notificación y vacíos en el conocimiento.

Conclusión: La síntesis de estos factores puede utilizarse para optimizar medidas asistenciales y de gestión, proporcionando recursos materiales, personales, capacitación y promoción de la cultura de seguridad, con el fin de incentivar la notificación y buscar indicadores fidedignos de estos agravios.

\section{Introdução}

A segurança do paciente é tema gerador de discussão ao identificar a ocorrência de eventos adversos (EA) e propor melhorias para a qualidade na assistência em saúde. ${ }^{(1)}$ Reconhecendo que EA são danos ao paciente não relacionados à evolução natural da doença, percebe-se como necessárias açôes de controle e prevenção, frente ao potencial de agravos à recuperaçáo do paciente. ${ }^{(2)}$ Embora seja atribuída maior ênfase ao ambiente hospitalar, a abordagem da segurança do paciente está em contínua expansão, direcionando-se às diversas vertentes e sistemas de saúde. ${ }^{(3)}$

Considera-se que a qualidade da assistência está atrelada à identificação de EA, com o foco de detectar falhas sistêmicas, e não a punição pessoal, haja vista que erros, embora praticados de modo individual, podem decorrer de falhas estruturais ou processuais do sistema ou organização de saúde. ${ }^{(4)}$ Conhecer fragilidades e falhas sistêmicas que resultam em EA oportuniza o planejamento de açóes preventivas. Deste modo, destaca-se a relevância do sistema de notificação no processo de melhoria contínua da assistência e relativa à segurança do paciente.

A prática da notificação está respaldada na legislação brasileira, a qual solicita detalhamento das características do EA por meio da ferramenta eletrônica NOTIVISA. ${ }^{(5)}$ Segundo relatório do Ministério da Saúde (MS), o Paraná, é o terceiro estado que mais notifica incidentes em número absoluto, com aproximadamente 20 mil casos no ano de 2019. No Brasil, entre os períodos de julho a dezembro de 2019, os principais incidentes notificados foram relativos à falhas durante a assistência em saúde, lesão por pressão e falhas envolvendo cateter venoso. ${ }^{(6)}$

Estudo transversal, realizado em hospital geral, identificou que as unidades de internação e de terapia intensiva se destacavam entre os setores com maior registros de notificação espontânea; $71 \%$ comunicadas por enfermeiros, sendo frequentemente relacionadas ao processo de medicar e lesóes por pressão. ${ }^{(7)} \mathrm{A}$ subnotificação é reconhecida como empecilho para o planejamento de açóes direcionadas aos fatores geradores de erros sistêmicos. ${ }^{(8)}$ Logo, destaca-se a importância de investigar fatores que dificultam e facilitam a prática, de modo a promover esse sistema.

Diante do exposto, essa revisão teve como objetivo analisar as evidências disponíveis na literatura acerca dos fatores dificultadores e facilitadores para a notificação de eventos adversos.

\section{Métodos}

Trata-se de revisão integrativa da literatura, a qual é um método de prática baseada em evidência que 
permite aprofundamento de determinado tema pela síntese e análise de resultados dos estudos, para incorporação na prática clínica. $\mathrm{O}$ estudo seguiu seis etapas: formulação da questão norteadora, estabelecimento de critérios, categorização dos estudos primários, avaliação, interpretação e síntese. ${ }^{(9)}$

A questão norteadora "Quais os fatores facilitadores e dificultadores que interferem na notificação de eventos adversos em serviços de saúde?" foi embasada no acrônimo PCC - Problema: fatores que interferem na notificação de eventos adversos, Conceito: eventos adversos e Contexto: serviços de saúde.

Dois pesquisadores, de forma independente, fizeram a busca no mês de março de 2020, utilizando a estratégia com os descritores em Ciências da Saúde (DeCS) e operadores booleanos: "erros médicos" $O R$ "erros de medicação" $A N D$ "notificaçãa”, "errores médicos" OR "errores de medicación" $A N D$ "notificación" e "medical error" OR "medication errors" AND "notification" nas bases de dados Latin American \& Caribbean Health Sciences Literature (LILACS), Medical Literature Analysus and Retrieval System Online (MEDLINE) e Bibliográficas especializada na área de Enfermagem (BDENF) por meio do portal da Biblioteca Virtual de Saúde (BVS). $\mathrm{Na}$ National Library of Medicine (PubMed) aplicaram-se os descritores do Medical Subject Headings (MeSH), com a seguinte estratégia: "risk management" [Mesh] OR "patient safety" [Mesh] AND "mandatory reporting" [Mesh] e no Scopus "risk management" OR "patient safety" $A N D$ "mandatory reporting" (Quadro 1).

Quadro 1. Estratégias de busca para seleção dos estudos

\begin{tabular}{|l|l|l|}
\hline & Estratégia & $\begin{array}{l}\text { Bases de dados e } \\
\text { números dos estudos }\end{array}$ \\
\hline Estratégia 1 & $\begin{array}{l}\text { "erros médicos" OR "erros de medicação" } \\
\text { AND "notificação" }\end{array}$ & $\begin{array}{l}\text { MEDLINE (595), LILACS } \\
\text { (39) e BDENF (23) }\end{array}$ \\
\hline Estratégia 2 & $\begin{array}{l}\text { "errores médicos" OR "errores de medicación" } \\
\text { AND "notificación" }\end{array}$ & $\begin{array}{l}\text { MEDLINE (45), LILACS (25) } \\
\text { e BDENF (14) }\end{array}$ \\
\hline Estratégia 3 & $\begin{array}{l}\text { "medical erros" OR "medication errors" AND } \\
\text { "notification" }\end{array}$ & $\begin{array}{l}\text { MEDLINE (966), LILACS } \\
\text { (34) e BDENF (20) }\end{array}$ \\
\hline Estratégia 4 & $\begin{array}{l}\text { "risk management" [Mesh] OR "patient safety" } \\
\text { [Mesh] AND "mandatory reporting" [Mesh] }\end{array}$ & PubMed (453) \\
\hline Estratégia 5 & $\begin{array}{l}\text { "risk management" OR "patient safety" AND } \\
\text { "mandatory reporting" }\end{array}$ & Scopus (481) \\
\hline
\end{tabular}

Os critérios de inclusão foram: artigos primários publicados de janeiro de 2015 a dezembro de 2019, nos idiomas português e/ou espanhol e/ou inglês. Foram excluídos estudos que não respondessem à questão norteadora. As 2.195 publicaçóes resultantes da busca foram armazenadas e organizadas com auxílio da ferramenta EndNote Basic online, a qual removeu aquelas duplicadas. Inicialmente foram aplicados os filtros e realizada a leitura dos títulos e resumos utilizando os critérios de inclusão e exclusão. Foram selecionados 31 artigos para leitura na íntegra, dos quais 20 não responderam à questáo norteadora e 11 foram eleitos para a síntese da revisão. O processo de seleção dos estudos foi apresentado por meio do fluxograma The Preferred Items for Systematic Reviews and Meta-Analysis (PRISMA) ${ }^{(10)}$ (Figura 1).

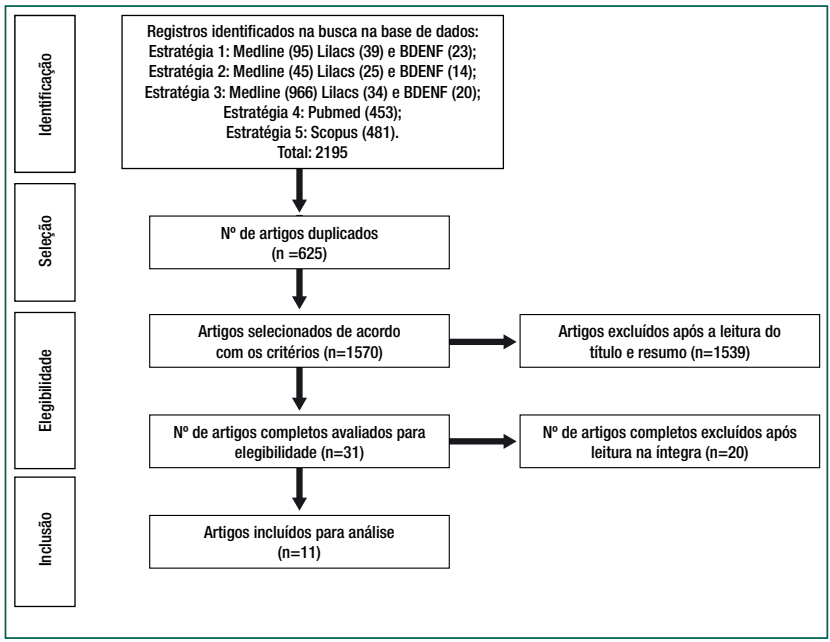

Figura 1. Fluxograma do processo de seleção dos estudos

Os estudos selecionados, num primeiro momento, foram analisados por meio de um roteiro desenvolvido pelos autores contendo: autores, país, ano de publicação, título, objetivo e nível de evidência. Num segundo momento os estudos foram agrupados por similaridade, elencando os fatores facilitadores e dificultadores da notificação de EA. Os artigos foram classificados segundo o nível de evidência, de A (maior evidência) a D (menos evidência) de acordo com os parâmetros do Oxford Centre for Evidence-based Medicine. ${ }^{(11)} \mathrm{A}$ interpretação e síntese do conhecimento, para incorporação na prática clínica, foram apresentadas de forma descritiva e tabular. 


\section{Resultados}

Os artigos elegidos foram apresentados no quadro 2, com informaçóes relativas a autores, ano, país de publicação, título, objetivos e nível de evidência.

Os fatores facilitadores e dificultadores para a notificação de EA, e correspondentes artigos, foram apresentados de modo agrupado no quadro 3 .

\section{Discussão}

Os fatores foram agrupados para a organização e síntese, resultando em 10 facilitadores, apontados 28 vezes, e 10 dificultadores, apontados 39 vezes nos artigos. Todos os estudos apresentaram, pelo menos, um as- pecto facilitador e dificultador para a notificação de EA. A quantidade de fatores facilitadores evidencia que o sistema de notificação não é incipiente, ${ }^{(22)}$ como demonstrado nos estudos analisados nesta revisão.

$\mathrm{O}$ apoio institucional aos profissionais foi o fator mais frequentemente apontado nas produçóes científicas, e abrange a garantia da confidencialidade do relato do EA, ressaltada em estudo semelhante, assim como o sentimento, pelo profissional notificador, de que será amparado pelos colegas e instituição. ${ }^{(23)}$

A cultura de segurança organizacional também foi destacada. Considera-se haver forte correlação entre a prestação do cuidado seguro e a cultura da segurança, ${ }^{(24)}$ sendo que esta afeta o ato de notificar, já que há reconhecimento da subnotificação, tanto no Brasil como em outros países. ${ }^{(25)}$ Como as notificaçôes são impres-

Quadro 2. Caracterização dos artigos elegidos para análise

\begin{tabular}{|c|c|c|c|c|}
\hline $\mathrm{N}^{\circ}$ & Autores, país e ano de publicação & Título & Objetivo & $\begin{array}{l}\text { Nível de } \\
\text { evidência }\end{array}$ \\
\hline 1 & $\begin{array}{l}\text { Souza VS; Kawamoto AM; Oliveira JL; Tonini NS. } \\
\text { Brasil, 2015.(12) }\end{array}$ & $\begin{array}{l}\text { Errors and adverse events: The interface with health } \\
\text { professionals' safety culture. }\end{array}$ & $\begin{array}{l}\text { Analisar a cultura de segurança em relação aos eventos } \\
\text { adversos. }\end{array}$ & $2 \mathrm{~B}$ \\
\hline 2 & $\begin{array}{l}\text { Koehn AR; Ebright PR; Draucker. Estados Unidos, } \\
\text { 2016. }{ }^{(13)}\end{array}$ & Nurses' experiences with errors in nursing. & Explorar a tomada de decisão em relatar erros médicos. & $2 \mathrm{C}$ \\
\hline 3 & $\begin{array}{l}\text { Jafree SR; Zakar R; Zakar MZ; Fischer F. } \\
\text { Paquistão, 2016. }{ }^{(14)}\end{array}$ & $\begin{array}{l}\text { Nurse perception of organizational culture and its } \\
\text { association with the culture of error reporting: a case of } \\
\text { public sector hospital in Pakistan. }\end{array}$ & $\begin{array}{l}\text { Investigar a associação entre a cultura organizacional e a } \\
\text { cultura de notificação. }\end{array}$ & $2 \mathrm{~B}$ \\
\hline 4 & $\begin{array}{l}\text { Duarte SC; Bessa AT; Buscher A; Stipp MA. } \\
\text { Brasil, 2016. }{ }^{(15)}\end{array}$ & Error characterization in intensive care nursing. & $\begin{array}{l}\text { Identificar e discutir erros de acordo com a teoria do erro } \\
\text { humano. }\end{array}$ & $2 \mathrm{~B}$ \\
\hline 5 & $\begin{array}{l}\text { Lanzilloti L S; Andrade CL; Mendes W; Seta MH. } \\
\text { Brasil, 2016. }{ }^{(16)}\end{array}$ & $\begin{array}{l}\text { Eventos adversos e incidentes sem dano em recém- } \\
\text { nascidos notificados no Brasil, nos anos } 2007 \text { a } 2013 .\end{array}$ & $\begin{array}{l}\text { Analisar os eventos adversos e outros incidentes sem } \\
\text { danos. }\end{array}$ & $2 \mathrm{~B}$ \\
\hline 6 & $\begin{array}{l}\text { Siman AG; Cunha GS; Brito MJ. } \\
\text { Brasil, 2017. }{ }^{(8)}\end{array}$ & $\begin{array}{l}\text { A prática de notificação de eventos adversos em um } \\
\text { hospital de ensino. }\end{array}$ & Compreender a prática de notificação. & C \\
\hline 7 & $\begin{array}{l}\text { Marinho MM; Radunz V; Rosa LM; Tourinho FSV; } \\
\text { llha P; Misiak. } \\
\text { Brasil, 2018. }{ }^{(17)}\end{array}$ & $\begin{array}{l}\text { Resultados de intervenções educativas sobre segurança } \\
\text { do paciente na notificação de erros e eventos adversos. }\end{array}$ & $\begin{array}{l}\text { Avaliar os resultados de intervenções educativas na } \\
\text { notificação. }\end{array}$ & $2 \mathrm{~B}$ \\
\hline 8 & $\begin{array}{l}\text { de Vos MS; Hamming JF; Hendriks JJ; Mheen PJ. } \\
\text { Holanda, 2019.(18) }\end{array}$ & $\begin{array}{l}\text { Connecting perspectives on quality and safety: patient-level } \\
\text { linkage of incident, adverse event and complaint data. }\end{array}$ & $\begin{array}{l}\text { Estabelecer relação entre eventos adversos e incidentes } \\
\text { com queixas. }\end{array}$ & $2 \mathrm{~B}$ \\
\hline 9 & $\begin{array}{l}\text { Pérez CD; Fuentes PS; García EJ. } \\
\text { Espanha, 2019.(19) }\end{array}$ & $\begin{array}{l}\text { Addressing medical errors: an intervention protocol for } \\
\text { nursing professionals. }\end{array}$ & $\begin{array}{l}\text { Identificar intervenções a serem incluídas em protocolo } \\
\text { frente à eventos adversos graves. }\end{array}$ & $2 \mathrm{~B}$ \\
\hline 10 & $\begin{array}{l}\text { Batista J; Cruz ED; Alpendre FT; Paixão DP; Gaspari } \\
\text { AP; Mauricio AB. } \\
\text { Brasil, 2019. }{ }^{(20)}\end{array}$ & $\begin{array}{l}\text { Cultura de segurança e comunicação sobre erros } \\
\text { cirúrgicos na perspectiva da equipe de saúde. }\end{array}$ & $\begin{array}{l}\text { Analisar a dimensão comunicação da cultura de } \\
\text { segurança e notificação. }\end{array}$ & $2 \mathrm{~B}$ \\
\hline 11 & $\begin{array}{l}\text { Mascarenhas FA; Anders J C; Gelbcke FL; Lanzoni } \\
\text { GM; Ilha P. } \\
\text { Brasil, 2019.(21) }\end{array}$ & $\begin{array}{l}\text { Facilidade e dificuldade dos profissionais de saúde frente } \\
\text { ao processo de notificação de eventos adversos. }\end{array}$ & $\begin{array}{l}\text { Descrever as facilidades e as dificuldades no processo de } \\
\text { notificação. }\end{array}$ & $2 \mathrm{~B}$ \\
\hline
\end{tabular}

Quadro 3. Fatores dificultadores e facilitadores para a notificação de eventos adversos

\begin{tabular}{|l|l|l|l|}
\hline Fatores facilitadores & Artigos & Fatores dificultadores & Artigos \\
\hline Apoio institucional aos profissionais. & $1,2,3,4,6,9$ & Falta de recursos materiais/humanos. & $5,6,7,8,9,10,11$ \\
\hline Cultura de segurança organizacional. & $3,5,7,8,10$ & Medo/vergonha. & $1,2,6,7,10,11$ \\
\hline Aprimoramento do sistema de notificação. & $2,5,7,8$ & Postura institucional punitiva/falta de amparo. & $1,2,4,5,7,9$ \\
\hline Incentivo ao relato voluntário e confidencial. & $2,6,7,9$ & Falta de estímulo à notificação. & $2,3,4,8,9,11$ \\
\hline Atividades educativas. & $1,7,9$ & Lacunas no conhecimento. & $6,7,9,11$ \\
\hline Inclusão de pacientes e familiares, e uso de queixas como gatilhos para reconhecer eventos adversos. & 2,11 & Falhas na comunicação. & $1,2,10$ \\
\hline Envolvimento dos profissionais responsáveis pela assistência direta no planejamento de ações de segurança. & 1 & Decisão de não notificar por julgamento pessoal. & 2,7 \\
\hline Indicadores para monitoramento de eventos adversos. & 2 & Ausência de sistema formal. & 3,11 \\
\hline Sistematização da assistência para mudanças na cultura. & 3 & Relação ruim entre líderes e liderados. \\
\hline Participação da alta liderança. & 7 & Notificação restrita aos enfermeiros. \\
\hline
\end{tabular}


cindíveis para o cálculo das taxas de EA, e a produção de indicadores da qualidade do serviço prestado, a subnotificação implica em dados não confiáveis sobre a prestação de cuidado seguro e da qualidade da assitencial, prejudicando a construção de estratégias para a melhoria do cuidado e da segurança do paciente. ${ }^{(24)}$

A notificação espontânea, por meio do preenchimento de fichas, impressas ou eletrônicas, é o principal meio para relato e considerado de baixo custo, contudo, requer envolvimento dos profissionais assistenciais, administrativos e de apoio. ${ }^{(7)}$ Trata-se de um sistema com co-participatição, o qual dependente da motivação individual e coletiva, sujeito às mudanças gerenciais e a cultura de segurança institucional.

As atividades educativas sistemáticas minimizam a falta de conhecimento sobre que tipo de incidente caracterizado, como, por exemplo, erro no preparo ou administração de medicamentos, e mesmo dúvidas de como realizar a notificação. ${ }^{(25)}$ Enfermeiros relatam dificuldades ao utilizar meios para notificação, e desconhecimento do fluxo, demonstrando que aprimorar os sistemas e buscar outros métodos de buscas de EA, de fato, pode contribuir para a melhora na comunicação e detecção desses agravos. ${ }^{(26)}$

Um dos fatores facilitadores apresentados foi a inclusão de pacientes e familiares, e o uso de suas queixas como gatilhos para rastrear eventos. $\mathrm{O}$ fato de estar presente em dois $(18,1 \%)$ dos estudos reflete que, embora esta abordagem não seja comum, a participação do paciente e seus familiares no reconhecimento de EA é inovador. Tanto que instrumentos de identificação consideram as queixas como gatilho para a quantificação da ocorrência e reconhecimento dos agravos. ${ }^{(27)} \mathrm{O}$ estímulo à participação do paciente, na própria segurança, e dos familiares, faz parte das estratégias de gestão de segurança do paciente. ${ }^{(28)}$

Quatro fatores facilitadores foram citados uma única vez, a saber, o envolvimento dos profissionais responsáveis pela assistência direta no planejamento de açóes de segurança; o emprego de indicadores para monitoramento dos eventos; a sistematização da assistência para promover mudanças na cultura e a participação da alta liderança. Por estarem relacionados ao desenvolvimento da cultura de segurança, esses potencialmente favorecem a notificação, no sentido de que a cada evento náo notificado perde- -se uma oportunidade para a correção das possíveis falhas do sistema. ${ }^{(7)}$ Destaca-se que nas instituiçôes onde a Sistematização da Assistência de Enfermagem está estabelecida, os cuidados relativos aos seis protocolos de segurança do paciente, preconizados pelo Ministério da Saúde brasileiro, ${ }^{(29)}$ integram a prescrição de enfermagem. Deste modo, EA relativos a esses são registrados em prontuário, favorecendo a identificação dos agravos e as correspondentes notificaçóes.

Dentre os fatores dificultadores, a falta de recursos materiais e humanos foi a mais apontada, sendo citada em sete dos 11 artigos analisados. Esse fator vem ao encontro de estudo no qual profissionais de enfermagem declaram haver número insuficiente de profissionais de saúde, com consequente sobrecarga de atividades ${ }^{(30)}$ fator que implica em subnotificação, uma vez que esse registro pode náo ser percebido como prioritária frente aos cuidados diretos ao paciente.

Um ponto importante identificado foi o medo ou vergonha, por parte dos profissionais, o que corrobora com outro fator apontado, que corresponde à postura institucional punitiva/falta de amparo. Fatos esses que refletem precária cultura de segurança, quando a organização não ampara os profissionais e não os vê como segundas vítimas do sistema. ${ }^{(8)} \mathrm{O}$ medo, fator dificultador mais citado, também está relacionado à qualidade da relação hierárquica entre a liderança e seus subordinados.

Em estudo que objetivou identificar motivos para a não notificação, $70,1 \%$ dos profissionais relataram que alguns erros de medicação não são notificados por medo da reaçáo dos enfermeiros responsáveis. ${ }^{(25)}$ Deste modo, a subnotificação pode, também, estar associada à postura da liderança frente ao erro, sendo desejável atitude inclusiva, de apoio e corretiva, e não intimidadora, a qual propicia a ocultação do EA. A equipe de enfermagem reconhece que o relato desses eventos, especialmente os mais graves, são importantes e tem consequências, mas anseiam pelo respaldo dos enfermeiros e, quando isso não acontece, deixam de notificar. ${ }^{(21)}$

A constatação de subnotificação geralmente tem por origem a postura institucional punitiva, fator dificultador citado em seis artigos, reforçando que o medo pode interferir no julgamento pessoal de notificar, ou não. ${ }^{(30)}$ Mudança na abordagem, de punitiva para 
educativa, também é aplicável ao ambiente de ensino formal, no qual a postura do professor influencia, de modo importante, a atitude do aluno frente ao EA.

Lacunas no conhecimento sobre o tema, ou de como realizar notificaçôes, também foram citadas como razão para a não notificação, demonstrando haver necessidade de investimento educativo do que caracteriza o EA, de como e onde notificar. ${ }^{(25)} \mathrm{Em}$ contrapartida, o registro e a investigação dos eventos contribuem para direcionar práticas de educação continuada, retroalimentar e qualificar a assistência. (24) Neste contexto, falhas na comunicação são frequentes na área de saúde, considerada sistema complexo com múltiplos atores interagindo simultaneamente. ${ }^{(31)}$ A notificação é percebida como um meio de comunicação que alerta a instituição sobre falhas e erros, e proporciona a criação de banco de dados norteador para o planejamento de açóes preventivas e prestação de cuidados mais seguros. ${ }^{(21)}$

Nesse processo de comunicação, o feedback tem papel relevante como estímulo à notificaçóes. O retorno ao notificador fortalece o carater não punitivo, o ambiente de aprendizagem com o erro e, principalmente, possibilita a reflexão sobre a assistência prestada e fatores que influenciam a ocorrência do erro. Deste modo, essa é estratégia que propicia a reflexão e o sentimento de fazer parte da soluçáo, fundamental às ações preventivas. Esses fatores podem reverter a decisão de não notificar por julgamento pessoal, outro dos fatores dificultadores apontados neste estudo. ${ }^{(21)}$

Ao receber o feedback da notificação, o profissional tem a oportunidade de atribuir maior valor a essa prática, ou seja, perceber que sua atitude foi reconhecida e é importante para a organização. Considerando a força e a característica do trabalho desenvolvido, o enfermeiro é profissional-chave nos sistemas de notificação, tanto no Brasil como em outros países. São os que mais notificam e geralmente formalizam as notificaçóes originadas da assistência de outras categorias profissionais. ${ }^{(7)}$ Infere-se que, por outro lado, a natureza de sua atividade, caracterizada pelo cuidado direto e ininterrupto ao paciente, seja um fator dificultador frente à sobrecarga de trabalho, além das múltiplas responsabilidades.

A adesão à notificação por outros profissionais também pode ser aprimorada com o uso de sistemas formais (institucionais), simples e que ocupem pouco do tempo já escasso para a assistência. ${ }^{(26)}$

A maioria dos artigos analisados demonstraram bom nível de evidência (2B), correspondendo a estudos de coorte, agregando maior confiabilidade aos resultados desta análise.

Entre as limitaçôes deste estudo, apontamos o recorte temporal e as estratégias de busca que podem ter cerceado a identificação de mais publicaçôes. Dentre as oportunidades para o aprofundamento do tema desta pesquisa, e a partir dos fatores dificultadores à notificação de eventos identificados na literatura, aponta-se a necessidade de explorar, principalmente, os sentimentos de medo e vergonha dos profissionais e sua relação como os aspectos punitivo e de falta de apoio e estímulo institucional, por serem modificáveis e fortemente associados à cultura de segurança institucional. Ainda, considerando-se a relevância do conhecimento relativo do porquê, como e quando notificar, esta lacuna, embora não identificada com maior frequência nos estudos, indica importante aspecto a ser explorado no âmbito da formação e da prática profissional em saúde.

\section{Conclusão}

Carência de ações preventivas à ocorrência de EA; falta de recursos humanos e materiais e de estímulo à notificação, associados ao medo em notificar foram os principais fatores dificultadores identificados no estudo, devendo esses serem reconhecidos como barreiras a identificação dos problemas sistêmicos associados à ocorrência desses agravos. Recomenda-se açóes para minimizar barreiras institucionais e comportamentais que dificultam a notificação de EA, e a utilização da educação continuada como instrumento para sua promoção. Da mesma forma, o fortalecimento dos fatores facilitadores identificados pode contribuir à robustez do sistema de notificação, tendo por foco o aprendizado com o erro. Espera-se que a síntese dos principais fatores identificados possa ser utilizada em pesquisas e medidas assistenciais e gerenciais, com vistas à qualidade e fidedignidade dos dados, subsidiando o planejamento de açóes direcionadas e, possivelmente, mais assertivas que resultem na proteção dos pacientes. 


\section{Agradecimentos}

\section{Esse estudo foi realizado com o apoio da Coordenação de Aperfeiçoamento de Pessoal de Nível Superior - Brasil (CAPES) - código de financiamento 001 e pelo Conselho Nacional de Desenvolvimento Científico e Tecnológico (CNPq).}

\section{Referências}

1. Oliveira AC, Garcia PC, Nogueira LD. Nursing workload and occurrence of adverse events in intensive care: a systematic review. Rev Esc Enferm USP. 2016;50(4):683-94.

2. World Health Organization (WHO). The Conceptual Framework for the International Classification for Patient Safety. Version 1.1. Final Technical Report. Geneva: WH0; 2009 [cited 2020 Apr 6]. Available from: www.who.int/patientsafety/taxonomy/icps_full_report.pdf

3. Rodrigues CC, Santos VE, Sousa P. Patient safety and nursing: interface with stress and Burnout Syndrome. Rev Bras Enferm. 2017;70(5):1083-8.

4. Dutra, DD, Duarte MC, Albuquerque KF, Santos JS, Simões KM, Araruna PC. Adverse events in Intensive Care Units: bibliometric study. J Res Fundam Care Online. 2017;9(3):669-75.

5. Brasil. Agência Nacional de Vigilância Sanitária (ANVISA). Implantação do núcleo de segurança do paciente em serviços de saúde. Brasília (DF): ANVISA; 2016 [citado 2019 Set 20]. [Série Segurança do Paciente e Qualidade em Serviços de Saúde]. Disponível em: https:// www20.anvisa.gov.br/segurancadopaciente/index.php/publicacoes/ item/caderno-6-implantacao-do-nucleo-de-seguranca-do-paciente

6. Brasil. Agência Nacional de Vigilância Sanitária (ANVISA). Relatórios dos Estados - Eventos Adversos - Arquivos. Brasília (DF): ANVISA; 2020 [citado 2020 Abr 9]. Disponível em: www20.anvisa.gov.br/segurancadopaciente/ index.php/publicacoes/category/relatorios-dos-estados

7. Furini AC, Nunes AA, Dallora ME. Notifications of adverse events: characterization of the events that occurred in a hospital complex. Rev Gaúcha Enferm. 2019;40(Spe):e20180317.

8. Siman AG, Cunha SG, Brito MJ. The practice of reporting adverse events in a teaching hospital. Rev Esc Enferm USP. 2017;51:e03243.

9. Mendes KD, Silveira RC, Galvão CM. Revisão integrativa: método de pesquisa para a incorporação de evidências na saúde e na enfermagem. Texto Contexto Enferm. 2008;17(4):758-64.

10. Moher D, Liberati A, Tetzlaff J, Altman DG; PRISMA Group. Preferred reporting items for systematic reviews and meta-analyses: the PRISMA statement. PLoS Med. 2009;6(7):e1000097.

11. Comissão Nacional de Incorporação de Tecnologias no Sistema Único de Saúde (CONITEC). Nível de Evidência Científica por Tipo de Estudo "Oxford Centre for Evidence-based Medicine". CONITEC; 2009 [citado 2020 Abr 9]. Disponível em: http://conitec.gov.br/images/Artigos_ Publicacoes/Oxford-Centre-for-Evidence-Based-Medicine.pdf

12. Souza VS, Kawamoto AM, Oliveira JL, Tonini NS, Fernandes LM, Nicola AL. Erros and adverse events: the interface with health professionals' safety culture. Cogitare Enferm. 2015;20(3);474-81.

13. Koehn AR, Ebright PR, Draucker CB. Nurses' experiences with errors in nursing. Nurs Outlook. 2016;64(6):566-574.
14. Jafree SR, Zakar R, Zakar MZ, Fischer F. Nurse perceptions of organizational culture and its association with the culture of error reporting: a case of public sector hospitals in Pakistan. BMC Health Serv Res. 2016;16:3.

15. Duarte SD, Bessa AT, Büscher A, Stipp MA. Error characterization in intensive care nursing. Cogitare Enferm. 2016;21:1-8.

16. Lanzillotti LD, Andrade CL, Mendes W, Seta MH. Eventos adversos e incidentes sem dano em recém-nascidos notificados no Brasil, nos anos 2007 a 2013. Cad Saúde Pública. 2016;32:e00100415.

17. Marinho MM, Radünz V, Rosa LM, Tourinho FS, Iha P, Misiak M. Resultados de intervenções educativas sobre segurança do paciente na notificação de erros e eventos adversos. Rev Baiana Enferm. 2018;32(1):1-12.

18. de Vos MS, Hamming JF, Chua-Hendriks JJ, Marang-van de Mheen PJ. Connecting perspectives on quality and safety: patient-level linkage of incident, adverse event and complaint data. BMJ Qual Saf. 2019;28(3):180-9.

19. Pérez CD, Fuentes PS, García EJ. Addressing medical errors: an intervention protocol for nursing professionals. Ver Esc Enferm USP. 2019;53:e03463.

20. Batista J, Cruz ED, Alpendre FT, Paixão DP, Gaspari AP, Mauricio AB. Cultura de segurança e comunicação sobre erros cirúrgicos na perspectiva da equipe de saúde. Rev Gaúcha Enferm. 2019;40(Spe):e20180192.

21. Mascarenhas FA, Anders JC, Gelbcke FL, Lanzoni GM, Iha P. Facilidade e dificuldade dos profissionais de saúde frente ao processo de notificação de eventos adversos. Texto Contexto Enferm. 2019;28:e20180040.

22. Maia CS, Freitas DR, Gallo LG, Araújo WN. Registry of adverse events related to health care that results in deaths in Brazil, 20142016. Epidemiol Serv Saude. 2018;27(2):e2017320.

23. Silva EK, Resende TC, Oliveira OD, Pallarés EC. Eventos adversos (EA): conhecimento dos profissionais de enfermagem de um município do interior da Amazônia brasileira. Rev Cient Facul Educ Meio Ambiente. 2018;9(2):686-91.

24. Lanzoni GM, Goularte AF, Koerich C, Reisdorfer E, Miotello M, Meirelles $\mathrm{BH}$. Adverse events and near misses in hospitalization units of a specialized hospital in cardiology. Rev Min Enferm. 2019;e-1184.

25. Alves MD, Carvalho DS, Albuquerque GS. Barriers to patient safety incident reporting by Brazilian health professionals: an integrative review. Cienc Saude Coletiva. 2019;24(8):2895-908.

26. Ferezin TP, Ramos D, Caldana G, Gabriel CS, Bernardes A. Análise da notificação de eventos adversos em hospitais acreditados. Cogitare Enferm. 2017;22(2):e49644.

27. Mendes W, Travassos C, Martins M, Marques PM. Adaptação dos instrumentos de avaliação de eventos adversos para uso em hospitais brasileiros. Rev Bras Epidemiol. 2008;11(1):55-66.

28. Macedo RS, Bohomol E. Organizational structure analysis of the Patient Safety Center in hospitals of the Sentinel Network. Rev Gaúcha Enferm. 2019;40(Esp):e20180264.

29. Brasil. Ministério da Saúde. Portaria $n^{0} 529$, de $1^{0}$ de abril de 2013. Institui 0 Programa Nacional de Segurança do Paciente (PNSP). Brasília (DF): Ministério da Saúde; 2013 [citado 2019 Set 20]. Disponível em: http://bvsms.saude. gov.br/bvs/saudelegis/gm/2013/prt0529_01_04_2013.html

30. Paiva MC, Popim RC, Melleiro MM, Tronchim DM, Lima SA, Juliani CM. Motivos da equipe de enfermagem para a notificação de eventos adversos. Rev Lat Am Enfermagem. 2014;22(5):747-54.

31. Berto AM, Uhlmann V0, Erdmann RH, Kawase PR. Acreditação hospitalar: uma complementaridade proveitosa para a gestão da produção. Sist Gestão. 2017;12(4):447-61. 